\title{
Les genres du discours dans l'ancienne rhétorique : listes, schémas et mode d'emploi, avec un exemple (le discours de Germanicus)
}

\section{Christine Noille}

\section{(2) OpenEdition Journals}

Édition électronique

URL : http://journals.openedition.org/rhetorique/337

DOI : 10.4000/rhetorique.337

ISSN : 2270-6909

Éditeur

UGA Éditions/Université Grenoble Alpes

Édition imprimée

ISBN : 978-2-84310-274-5

Référence électronique

Christine Noille, «Les genres du discours dans l'ancienne rhétorique : listes, schémas et mode d'emploi, avec un exemple (le discours de Germanicus) », Exercices de rhétorique [En ligne], 3 | 2014 mis en ligne le 18 juin 2014, consulté le 12 septembre 2020. URL : http://journals.openedition.org/ rhetorique/337 ; DOI : https://doi.org/10.4000/rhetorique.337

Ce document a été généré automatiquement le 12 septembre 2020.

\section{c) (i) (2)}

Les contenus de la revue Exercices de rhétorique sont mis à disposition selon les termes de la Licence Creative Commons Attribution - Pas d'Utilisation Commerciale - Partage dans les Mêmes Conditions 4.0 International. 


\title{
Les genres du discours dans l'ancienne rhétorique : listes, schémas et mode d'emploi, avec un exemple (le discours de Germanicus)
}

\author{
Christine Noille
}

\section{Les genres du discours, de quoi s'agit-il ?}

1 Dans l'équipe de recherche Rare-Rhétorique de l'Antiquité à la Révolution, nous avons entrepris de mettre à jour un outil complexe de l'ancienne rhétorique, à la fois omniprésent $\mathrm{du} \mathrm{XVI}^{\mathrm{e}}$ au XIX ${ }^{\mathrm{e}}$ siècle et complétement oblitéré dans la brève histoire de notre contemporanéité, à savoir les formes instituées du discours (et de l'écrit épistolaire ${ }^{1}$ ). Nous nous appuierons pour les présenter schématiquement sur deux articles publiés dans les numéros précédents de la revue Exercices de rhétorique, d'une part l'article que Francis Goyet a consacré à leur présentation dans son article «Le problème de la typologie des discours ${ }^{2}$ » et d'autre part les éléments que nous avons apportés dans notre essai de synthèse "Le discours d'un prince (Énéide I, 597-610). Archéologie de la disposition ${ }^{3} »$. Nous nous arrêterons sur quatre points.

\section{Les recenser}

2 Les listes des discours institués et spécifiés sont très stables sur trois siècles de rhétorique $\left(\mathrm{xvI}^{\mathrm{e}}-\mathrm{xIX}^{\mathrm{e}}\right.$ siècles $\left.^{4}\right)$ :

- relevant du démonstratif, les panégyriques et les blâmes, les épithalames et oraisons funèbre, les discours de remerciements, de félicitations et de lamentations, les allocutions de bienvenue, d'inauguration et de départ ; 
- relevant du délibératif, les discours d'admonestation et de recommandation, les discours d'union et de sédition, le conseil, la consultation, l'exhortation, la consolation, la requête (directe : petitio, ou médiate : intercessio) ;

- enfin relevant du judiciaire, les discours d'accusation et de défense, les invectives et les objurgations, les récriminations et les discours de rupture, les discours de menace et les prières.

\section{Les schématiser}

3 Comment sont décrits les discours institués dans les anciennes rhétoriques? Trois approches sont conviées: une approche par la situation de communication, une approche par le canevas type, une approche par les cas exemplaires.

- Spécifier un discours par la situation de communication tout d'abord ne suppose pas un pur repli sur l'analyse du contexte pragmatique. Car si des éléments tels que le but poursuivi, l'éthos du locuteur ou la position du destinataire, sont génériquement identitaires, il s'agit toujours de savoir comment ils sont construits dans et par les énoncés, comment l'argumentaire élabore tel éthos, se focalise sur telle proposition, énonce les raisons de tel ou tel pathos - avec, en toile de fond, des outils disponibles tels que les répertoires de pathos et de topoi pour chaque pathos, et, nous y revoilà, les répertoires de discours institués, qui listent à la fois l'ensemble des buts possibles ${ }^{5}$ et pour chacun des discours, un panel possible de positions éthiques et de ressorts pathétiques.

- Mais l'approche du discours par sa finalité argumentative et ses fonctionnalités éthiques et pathétiques laisse rapidement la place dans les rhétoriques à un développement plus long, à savoir le listing des énoncés types, à utiliser selon les divers cas possibles répertoriés et selon le moment dans le discours. Il s'agit donc là d'une description séquentielle selon un canevas type, modulable et dynamique. Chaque discours n'est pas seulement défini par la situation de communication qu'il modélise, mais par le séquençage syntagmatique du discours conçu comme un tout, en parties et en topoi, par sa division et son ordonnancement. Les genres du discours sont aussi des formes du discours.

- Enfin, pour chaque embranchement dans les possibles d'une fonction et les possibles d'une forme, sont mentionnées les références précises des passages textuels (le plus souvent antiques, parfois contemporains) qui en donnent une formulation exemplaire.

Autrement dit, les rhétoriques mobilisent et transmettent à la fois un répertoire des pratiques discursives institutionnelles, une encyclopédie des formes et une bibliothèque des exemples.

\section{Les nommer}

Comment nommer ces discours formalisés et institués ? Vaste question, dont les enjeux théoriques sont réels mais dont les conséquences pratiques sont somme toute assez minces.

$6 \quad$ Types ou genres? Nous dirons tout d'abord qu'entre un discours tel qu'il est défini, décrit et exemplifié dans une rhétorique, et tel cas attesté, le rapport est bien de modulation et d'hybridation, comme entre un idéal type et un token, une occurrence (d'où le choix de F. Goyet de les présenter sous le terme de "types» de discours). Nous pouvons cependant noter que la compréhension à la fois pragmatique et formelle de ces situations discursives standardisées est très strictement ce à quoi font référence 
Volochinov puis Bakhtine lorsqu'ils parlent des "genres» du discours ${ }^{6}$, à la fois en termes institutionnels (communicationnels: en fonction des positions respectives d'interlocution et du but discursif) et en termes formels (syntaxiques : en partant du tout vers les parties; et dynamiques: en rapport à la conduite et à la poursuite discursive). Ils rejoignent en cela précisément l'ancienne théorie des species orationis dont nous faisons ici l'archéologie, et sans plus nous étendre sur ce point qui demandera assurément qu'on y revienne plus longuement, disons que nous nous autoriserons alors de cette tradition au présent pour reprendre leur propre appellation et requalifier nos objets en " genres $d u$ discours ».

7 Genres du discours ou genres de discours? Ce faisant, nous les dissocierons également de ce que l'histoire littéraire nomme les trois genera dicendi ou "genres de discours", autrement dit les trois grandes catégories discursives englobantes (le démonstratif, le délibératif, le judiciaire). Nous ajouterons simplement qu'entre les genera dicendi et les species orationis, la différence n'est pas de l'ordre de la modulation ni de l'application: les premiers relèvent de postulats et les seconds de l'usus. Les premiers sont opératoires au niveau de la théorie; mais seuls les seconds interviennent dans la pratique, qu'il s'agisse de l'art d'écrire ou de l'art de lire. On lit les textes exemplaires en repérant dans le fil de leurs énoncés le ou les genres du discours qu'ils exemplifient, apprenant ainsi à les reconnaitre, avant que de les imiter. Les genres du discours sont d'abord des formes opératoires pour structurer la lecture et la production des textes discursifs ${ }^{7}$.

8 Formes du discours ou figures de pensées? Une complication survient ici, en raison même de l'identification des genres par un canevas séquentiel. Un exemple suffira à comprendre le problème : l'expostulatio (ou reproches envers un ami) de même que la comminatio (ou menace) sont des genres discursifs comprenant un certain nombre de séquences hiérarchisées entre elles, dont les séquences focales que sont les formulations proprement dites $\mathrm{du}$ reproche ou de la menace. Mais ces mêmes formulations peuvent alors figurer à titre de séquences auxiliaires dans le canevas d'un autre genre, l'objurgation. Autrement dit, une comminatio est à la fois un genre du discours et une partie. Et à ce titre, une continuité syntagmatique existe entre un certain nombre de parties des discours et les figures de pensées: c'est ainsi que la menace et la prière sont mises en série avec d'autres formes, l'imprécation, l'optation, le serment, la promesse, la dubitation, la licence, etc., pour constituer la liste des figures de pensées ${ }^{8}$. Car dans la mesure où la figure de pensées ne relève pas uniquement d'un fonctionnement sémiotique, mais possède une assise matérielle, une extension syntagmatique, et qu'elle est à proprement parler une figure, une forme qui modèle et délimite un lieu dans le fil discursif', elle est susceptible d'isoler un bloc d'énoncé, une séquence, au même titre que les séquences discursives dont nous parlons ici. Nous dirons qu'entre la figure de pensées et la séquence mise en forme dans un canevas discursif, la différence n'est pas d'ordre syntagmatique mais fonctionnel : la séquence discursive joue un rôle dans l'édifice général de la partition discursive, contrairement à la figure de pensées, qui n'a qu'une incidence locale.

\section{Et maintenant, que fait-on?}

9 Nous nous sommes dotés des bases : une liste, des schémas, des noms. Voilà qui suffit à faire exister des catégories opératoires susceptibles de décrire quelque chose des discours. Mais on est loin encore de l'usus, de cet usage expert qui structure le rapport 
au discours tant sous l'angle de sa fabrique que de son appréciation. Et maintenant, que fait-on $^{10}$ ? En droit, nous pourrions nous lancer dans une tâche archéologique et récapitulative minutieuse, qui consisterait à mettre en parallèle les listes de canevas types, les schémas fonctionnels, les référents exemplaires et en tester la praticabilité et la rentabilité. Autant dire une tâche qui excède quelque peu les dimensions du présent article. Et il n'est pas sûr au demeurant qu'en commençant par là, on ait fait l'essentiel : dire l'intérêt de cet outillage pour construire des lisibilités nouvelles sur des textes.

Autant dire que nous ferons très strictement l'inverse : en nous focalisant sur un cas particulier, nous essaierons de voir comment les genres du discours permettent de décrire quelque chose $d u$ texte $^{11}$. Ou pour le dire autrement, nous essaierons de montrer comment les formes du discours et les genres qu'elles schématisent peuvent introduire un principe de lisibilité opératoire, en proposant sur le sémantisme du texte, des hypothèses de mise en forme et de focalisation.

11 Nous mènerons alors une expérience de lecture, à double fond: nous nous proposons de faire l'analyse rhétorique d'un discours, la harangue de Germanicus aux légions séditieuses ${ }^{12}$; et nous utiliserons pour ce faire le relais technique (pédagogique) de deux analyses rhétoriques anciennes déjà citées en note 4 , celles de Vossius $\left(1621^{13}\right)$ et de Pelletier $\left(1641^{14}\right)$.

Nous ajouterons deux précisions.

13 Première précision, méthodologique, il ne s'agira pas ici de faire l'archéologie d'une expérience de lecture érudite et de construire un savoir historique sur cette pratique particulière que serait l'ancienne rhétorique. Il n'est d'ancienne rhétorique que maintenue à distance comme objet d'un savoir ; à partir du moment où l'on bascule du côté des savoir-faire, on entre dans l'actualité d'une pratique méthodique.

Et précisément nous allons entrer de plain-pied dans la pédagogie de gestes techniques qui permettent, nous le verrons, des mises en forme concurrentes du texte en question; nous allons expérimenter ces gestes pour à notre tour produire une analyse concurrente et essayer au bout du compte de tirer quelques leçons de ce parcours. C'est donc une honnête proposition, de mener ensemble une expérience de lecture rhétorique sur un discours identifié, une expérience de formalisation rhétorique d'un discours.

Où l'on verra, seconde précision tout aussi méthodologique, que l'analyse rhétorique d'un texte procède par enquête sur des procédés standard - qu'elle suppose, teste, confirme ou reformule -, elle procède par sérialisation de l'énoncé dans une forme identitaire commune à d'autres exemples, les figures, les arguments types, les genres du discours - et se situe donc irréductiblement du côté de la théorie même si elle est en même temps dans la pratique des textes. Elle s'attache à identifier en eux le transversal ou l'exemplaire, l'architexte, et abandonne par conséquent à la " critique » au sens genettien du terme, tout discours sur ce qui en eux serait du côté du singulier et de l'irréductible.

\section{Le discours de Germanicus : herméneutique des contextes}

16 Venons-en au discours de Germanicus face aux légions séditieuses. À la façon de Queneau dans Les Fleurs bleues, arrêtons-nous un instant « pour considérer, un tantinet 
soit peu, la situation historique » : "Elle était plutôt floue ${ }^{15}$ ». Pour aller très vite, dans un côté du tableau, il y a la rébellion des légions romaines de Germanie en l'an 14 de notre ère ; et de l'autre côté, le général en chef, Germanicus, parvient à éteindre la sédition en trois temps.

Tout d'abord, Germanicus tente un premier discours, dont Tacite donne le synopsis au style indirect :

[TACITE. LE $1^{\text {er }}$ DISCOURS DE GERMANICUS] Tout le monde était assemblé pêlemêle: il leur ordonne de se ranger par manipules, afin de mieux entendre sa réponse; de prendre leurs enseignes, afin qu'il pût au moins distinguer les cohortes. On obéit, mais lentement. Alors, commençant par rendre un pieux hommage à Auguste, il passe aux victoires et aux triomphes de Tibère, et célèbre avant tout ses glorieuses campagnes en Germanie, à la tête de ces mêmes légions. Il leur montre l'accord unanime de l'Italie, la fidélité des Gaules, enfin la paix et l'union régnant dans tout l'empire. Ces paroles furent écoutées en silence ou n'excitèrent que des murmures. Mais lorsque, arrivé à la sédition, il leur demanda ce qu'était devenue la subordination militaire, où était la discipline qui leur faisait honneur autrefois, ce qu'ils avaient fait des centurions, des tribuns ; alors [...] ils lui reprochent à leur tour les cicatrices de leurs blessures, les traces de coups de verges. Bientôt des milliers de voix accusent ${ }^{16}[. .$.$] .$

18 Nous ne l'étudierons pas en détail mais il convient de mentionner qu'un rhétoricien le fera : en l'occurrence Vossius y voit le paradigme de ce que la rhétorique nomme la conciliatio, ce discours de la "réconciliation", discours qui, relevant de la scène politique et du délibératif, propose l'apaisement aux séditieux. De fait, Vossius commence par dresser le canevas type de la conciliatio avant d'en venir à cet exemple pour lui exemplaire.

Qu'est-ce qu'une conciliatio? Voici sa formalisation rhétorique standardisée : 


\section{VOSSIUS. LA CONCILIATIO OU DISCOURS DE RÉCONCILIATION ${ }^{17}$}

Pour réconcilier des partis qui s'affrontent, voici comment procéder. Nous montrons que toute offense doit être effacée : d'une part parce que c'est plus utile et plus sûr, et d'autre part, parce que c'est aussi plus honorable. Les passions qui règnent ici sont l'espoir et la crainte.

Mais autant il est facile d'exciter les gens à la sédition, autant il est difficile de les réconcilier. Car la plupart des gens sont trop attachés à leur colère et au souvenir de l'offense reçue. Là où il est le plus facile de ramener l'union, c'est face à une foule excitée. Alors peu importe que le discours soit éloquent, ce qui compte pour l'orateur est la protection d'un double bouclier, à savoir sa popularité et sa supériorité hiérarchique. [...] De fait, Jules César calma son armée en appelant ses hommes «Citoyens!» au lieu de "Soldats!»; et par ce seul mot, il leur fit faire volte-face et changer d'humeur (flexit), si bien qu'ils répondirent qu'ils étaient des soldats, et quoiqu'il leur contestât ce nom, ils le suivirent plus loin en Afrique. [...]

Cela posé, attention : il est très important que personne ne se risque à faire face immédiatement à une foule aveuglée par la colère, en s'imaginant protégé par son intégrité et son autorité. Car le premier mouvement d'une foule étant tout simplement belliqueux, ce n'est pas un discours qui vous permet de reprendre la main. En revanche, quand l'effervescence sera retombée, ce sera le moment propice pour leur parler. Mais d'abord il faudra établir le diagnostic sur l'origine de ce grand accès de fureur. Car le médecin qui ignore où est la plaie, comment y portera-t-il remède ? Le remède lui-même devra garder un certain équilibre : le discours commencera sur un ton amical, calmant; de là, on passera à des paroles de réprimande, plus sévères. Et dans l'application de l'un comme de l'autre remède, on respectera le dosage qu'exige la situation.

Germanicus ne l'a pas ignoré [...]. fois des éléments formels (concernant essentiellement ici les arguments types et le canevas type, l'inventio et la dispositio) et des éléments pragmatiques-discursifs (sur l'interprétation du contexte d'énonciation tel que postulé par le discours). Trois points sont à retenir ici car nous en aurons besoin :

21 1. le genre du discours de réconciliation s'insère dans une pragmatique de la prudence (il s'agit pour l'orateur de gérer le moment opportun où proposer la réconciliation; et il s'agit également de gérer le dosage du remède, avec un portrait du chef d'armée en médecin) ;

2. la conciliatio repose sur une argumentation contradictoire (alliance de séquences de réprimande et de séquences d'éloge, ou, au niveau des effets, ressorts de la crainte et de l'espoir) ;

3. enfin, le discours de réconciliation est relié à un canevas type qui a une pertinence dynamique, je veux dire qu'il est associé à un ordre syntagmatique dont le déroulement a une importance stratégique : d'abord le miel, ensuite l'amertume.

Disons alors, pour revenir au premier discours de Germanicus que Vossius analyse comme une conciliatio, que c'est un échec : peut-être le moment était-il mal choisi, peutêtre l'amertume était-elle mal administrée (on peut être sensible à la mollesse des reproches, sous la forme bien peu véhémente du questionnement...). Quoi qu'il en soit, c'est Germanicus qui se retrouve en position d'accusé, enseveli sous les reproches des 
séditieux. Ou comme l'écrit Vossius quand il modélise le texte sur le genre de la conciliatio :

[VOSSIUS. ANALYSE DU $1^{\text {er }}$ DISCOURS] [...] Germanicus ne l'a pas ignoré. Il commença par rendre un pieux hommage à Auguste, puis il passa aux victoires et aux triomphes de Tibère, en exaltant spécialement ses glorieuses campagnes en Germanie à la tête de ces légions-là.

Ensuite il exalta l'unanimité de l'Italie, la fidélité des Gaules, l'absence partout de troubles ou de désaccords.

Et puis, de là, insensiblement, il arriva à ces mots : «Où est la subordination militaire? Où est la discipline qui vous faisait honneur autrefois ? Qu'avez-vous fait de vos tribuns, de vos centurions?"

Cependant ce n'est pas avec ce discours qu'il parvint vraiment à les faire changer d'humeur ${ }^{18}[. .$.$] .$

Deuxième temps de cette reconquête de l'autorité, un acte politique réussi cette fois-ci, la fuite des épouses :

[TACITE. LE TABLEAU INTERMÉDIAIRE] On vit alors un départ déplorable, l'épouse d'un général fugitive et emportant son enfant dans ses bras, autour d'elle les femmes éplorées de leurs amis, qu'elle entraînait dans sa fuite, et, avec la douleur de ce triste cortège, la douleur non moins grande de ceux qui restaient. Ce tableau, qui annonçait plutôt une ville prise par l'ennemi que le camp et la fortune d'un César, ces pleurs, ces gémissements, attirèrent l'attention des soldats eux-mêmes. [...] Alors la honte (pudor) et la pitié (miseratio), le souvenir d'Agrippa son père, d'Auguste son aïeul, de son beau-père Drusus, l'heureuse fécondité d'Agrippine ellemême et sa vertu irréprochable, cet enfant né sous la tente [...], tout concourt à les émouvoir. Mais rien n'y contribua comme le dépit de se voir préférer les Trévires (invidia in Treviros ${ }^{19}$ ).

L'on notera au passage que Tacite lui-même en propose une analyse en termes d'affects et d'efficacité (est mis en avant le ressort du movere); analyse que reprendra littéralement la tradition rhétorique :

[VOSSIUS. ANALYSE DU TABLEAU] Ce que ni son éloquence d'homme ni son autorité n'avaient pu obtenir, se produisit avec le spectacle de son épouse, portant sur son sein leur fils encore tout petit, accompagnée des seules épouses de ses amis, quand en un cortège pitoyable toutes se réfugièrent chez les Trévires : la honte et la pitié (pudor et miseratio) d'une part, et d'autre part la jalousie envers les Trévires (invidia in Treviros), voilà ce qui arracha le revirement des soldats ${ }^{20}$.

Troisième temps enfin, on en arrive donc à la situation discursive à l'orée du discours qui va directement nous occuper, le second discours de Germanicus aux légions romaines, mis en valeur dans la prose tacitéenne par le passage au style direct et l'ampleur de son étendue. Avant de l'aborder, une brève récapitulation s'impose: le contexte large est certes celui de la sédition, mais le contexte étroit est désormais celui de la conversion. Avant même la seconde prise de parole de Germanicus, les soldats se sont rendus à de meilleurs sentiments. La preuve - textuelle - en est l'éthos des soldats avant et après le second discours, qui ne change pas: figé dans l'attitude du suppliant ${ }^{21}$. D'où une question tout à fait indécise : la situation est-elle bien encore celle de la conciliatio, de la délibération politique en vue d'en appeler à la réconciliation? Ou bien sommes-nous à l'étape suivante, et quelle est-elle? Autrement dit, quels sont les buts (les fonctions) de ce second discours?

Et pour s'en faire une première idée, le voici in extenso, avec son co-texte immédiat, mais sans les alinéas et paragraphages introduits par la tradition éditoriale - histoire d'être en prise avec une masse indifférenciée d'énoncé, sans formatage préétabli : 


\section{TACITE. LE SECOND DISCOURS DE GERMANICUS ${ }^{22}$}

Lui, encore ému de douleur et de colère, s'adressant à la foule qui l'environne : « Ne croyez pas, ditil, que mon épouse et mon fils me soient plus chers que mon père et la république. Mais mon père a pour sauvegarde sa propre majesté ; l'empire a ses autres armées. Ma femme et mes enfants, que j'immolerais volontiers à votre gloire, je les dérobe maintenant à votre fureur, afin que, si le crime ensanglante ces lieux, je sois la seule victime, et que le meurtre de l'arrière-petit-fils d'Auguste et de la belle-fille de Tibère n'en comble pas la mesure. En effet, qu'y a-t-il eu pendant ces derniers jours que n'ait violé votre audace? Quel nom donnerai-je à cette foule qui m'entoure? Vous appellerai-je soldats? Vous avez assiégé comme un ennemi le fils de votre empereur; citoyens? Vous foulez aux pieds l'autorité du sénat: les lois même de la guerre, le caractère sacré d'ambassadeur, le droit des gens, vous avez tout méconnu. Jules César apaisa d'un mot une sédition de son armée, en appelant Quirites des hommes qui trahissaient leurs serments. Auguste, d'un seul de ses regards, fit trembler les légions d'Actium. Si nous n'égalons pas encore ces héros, nous sommes leurs rejetons; et l'on verrait avec surprise et indignation le soldat d'Espagne ou de Syrie nous manquer de respect. Et c'est la première légion, tenant les enseignes de Tibère ; c'est vous, soldats de la vingtième, compagnons de ses victoires, riches de ses bienfaits, qui payez votre général d'une telle reconnaissance ! Voilà donc ce que j'annoncerai à mon père, qui de toutes les autres provinces ne reçoit que des nouvelles heureuses! Je lui dirai que ses jeunes soldats, que ses vétérans, ne se rassasient ni de congés, ni d'argent ; qu'ici seulement les centurions sont tués, les tribuns chassés, les députés prisonniers ; qu'ici le sang inonde les camps, rougit les fleuves ; qu'ici enfin ma vie est à la merci d'une multitude furieuse. Pourquoi, le premier jour où j'élevai la voix, m'arrachiez-vous le fer que j'allais me plonger dans le cœur, trop aveugles amis? Il me rendait un bien plus généreux office, celui qui m'offrait son glaive : j'aurais péri du moins avant d'avoir vu la honte de mon armée. Vous auriez choisi un autre chef, qui sans doute eût laissé ma mort impunie, mais qui eût vengé le massacre de varus et des trois légions. Car nous préservent les dieux de voir passer aux Belges, malgré l'empressement de leur zèle, l'éclatant honneur d'avoir soutenu la puissance romaine et abaissé l'orgueil de la Germanie ! Âme du divin Auguste, reçue au séjour des immortels, image de mon père Drusus, mémoire sacrée d'un grand homme, venez, avec ces mêmes soldats, sur qui la gloire et la vertu reprennent leurs droits, venez effacer une tache humiliante, et tournez à la ruine de l'ennemi ces fureurs domestiques. Et vous, dont je vois les visages, dont je vois les cœurs heureusement changés, si vous rendez au sénat ses députés, à l'empereur votre obéissance, à moi ma femme et mon fils, rompez avec la sédition, séparez de vous les artisans de trouble. Ce sera la marque d'un repentir durable, et le gage de votre fidélité.» Touchés par ce discours, ils lui demandent grâce, et, reconnaissant la vérité de ses reproches, ils le conjurent de punir le crime, de pardonner à l'erreur, et de les mener à l'ennemi [...].

\section{Première mise en forme possible : un discours de réconciliation}

Passons immédiatement à l'analyse rhétorique que Pelletier effectue de ce discours, car elle reprend l'outillage rhétorique que nous venons de mettre en place: Pelletier le modélise en effet sur la conciliatio telle que la décrit Vossius. C'est là une hypothèse à la fois formelle et fonctionnelle, qui lui permet de séquencer le texte et de mettre en réseau les séquences, autrement dit d'identifier des formes et de les insérer dans une cohérence, dans un système fonctionnel hiérarchisé.

Concernant l'opération de séquençage tout d'abord, dans la mesure où les commentaires de Pelletier alternent avec les citations littérales du texte tacitéen, nous 
nous trouvons de fait en prise avec une division du texte, lequel se trouve diffracté en quatre séquences successives dont les trois dernières sont identifiées par leur chapeau introducteur; tandis que le premier chapeau introduit quant à lui l'ensemble du discours mais ne dit rien de la première séquence, la laissant isolée (délimitée) mais non identifiée (non modélisée) : 


\section{PELLETIER. ANALYSE DU SECOND DISCOURS DE GERMANICUS : UNE CONCILIATIO ${ }^{23}$}

Magnifique discours [...] digne assurément d'être admiré comme spécimen éminent $d u$ discours de réconciliation:

Ne croyez pas, dit-il, que mon épouse et mon fils me soient plus chers que mon père et la république. Mais mon père a pour sauvegarde sa propre majesté ; l'empire a ses autres armées. Ma femme et mes enfants, que j'immolerais volontiers à votre gloire, je les dérobe maintenant à votre fureur, afin que, si le crime ensanglante ces lieux, je sois la seule victime, et que le meurtre de l'arrière-petit-fils d'Auguste et de la belle-fille de Tibère n'en comble pas la mesure. En effet, qu'y at-il eu pendant ces derniers jours que n'ait violé votre audace? Quel nom donnerai-je à cette foule qui m'entoure? Vous appellerai-je soldats? Vous avez assiégé comme un ennemi le fils de votre empereur; citoyens? Vous foulez aux pieds l'autorité du sénat: les lois même de la guerre, le caractère sacré d'ambassadeur, le droit des gens, vous avez tout méconnu. Jules César apaisa d'un mot une sédition de son armée, en appelant Quirites des hommes qui trahissaient leurs serments. [DESUNT : Auguste, d'un seul de ses regards, fit trembler les légions d'Actium. Si nous n'égalons pas encore ces héros, nous sommes leurs rejetons ; et l'on verrait avec surprise et indignation le soldat d'Espagne ou de Syrie nous manquer de respect].

Il rappelle ensuite les triomphes et les victoires de Tibère, pour leur reprocher leur attitude présente avec un ton d'autant plus sévère et direct, en tirant argument du genre de crime et des circonstances :

Et c'est la première légion, tenant les enseignes de Tibère; c'est vous, soldats de la vingtième, compagnons de ses victoires, riches de ses bienfaits, qui payez votre général d'une telle reconnaissance!

Il ajoute une menace en vue de susciter la crainte et la honte:

Voilà donc ce que j'annoncerai à mon père, qui de toutes les autres provinces ne reçoit que des nouvelles heureuses! Je lui dirai que ses jeunes soldats, que ses vétérans, ne se rassasient ni de congés, ni d'argent; qu'ici seulement les centurions sont tués, les tribuns chassés, les députés prisonniers ; qu'ici le sang inonde les camps, rougit les fleuves; qu'ici enfin ma vie est à la merci d'une multitude furieuse. Pourquoi, le premier jour où j'élevai la voix, m'arrachiez-vous le fer que j'allais me plonger dans le cœur, trop aveugles amis ? Il me rendait un bien plus généreux office, celui qui m'offrait son glaive : j'aurais péri du moins avant d'avoir vu la honte de mon armée. Vous auriez choisi un autre chef, qui sans doute eût laissé ma mort impunie, mais qui eût vengé le massacre de Varus et des trois légions. Car nous préservent les dieux de voir passer aux Belges, malgré l'empressement de leur zèle, l'éclatant honneur d'avoir soutenu la puissance romaine et abaissé l'orgueil de la Germanie!

Puis il met en avant la vénération d'Auguste et il frappe les esprits des soldats en recourant à un sentiment plus solennel d'espoir et d'amour:

Âme du divin Auguste, reçue au séjour des immortels, image de mon père Drusus, mémoire sacrée d'un grand homme, venez, avec ces mêmes soldats, sur qui la gloire et la vertu reprennent leurs droits, venez effacer une tache humiliante, et tournez à la ruine de l'ennemi ces fureurs domestiques. Et vous, dont je vois les visages, dont je vois les cœurs heureusement changés, si vous rendez au sénat ses députés, à l'empereur votre obéissance, à moi ma femme et mon fils, rompez avec la sédition, séparez de vous les artisans de trouble. Ce sera la marque d'un repentir durable, et le gage de votre fidélité. 

premiers mots de la séquence textuelle) :

Avançons quelques points de commentaire.

Tout le début du texte (de fait, presque la première moitié) est donc isolé, mais il n'est pas commenté. À la place d'une description précise de ce passage, nous trouvons donc une proposition générale sur tout le discours : est formulé le genre du discours et par là-même nous est indiqué de rechercher ce qu'on appelle en termes de l'art son propositum, sa proposition "générale ", en l'occurrence l'énoncé donnant l'impératif de conciliatio (i.e. arrêter la sédition). Nous le trouvons alors formulé littéralement dans l'ultime séquence, qui devient ainsi la séquence focale : «[...] rompez avec la sédition, séparez de vous les artisans de trouble.»

Les trois autres séquences, modélisées sur des catégories rhétoriques, lui sont par conséquent subordonnées :

Pelletier identifie, en seconde place dans l'ordre du discours, une séquence d'amplification du reproche, avec identification d'arguments types (le genre du crime et les circonstances).

Puis vient une séquence de menace (rappelons que le comminatoire, la comminatio, relève de deux ordres d'outils, tantôt figure de pensées quand il a une extension locale, tantôt genre du discours quand il est co-extensif à tout le discours).

Enfin, Pelletier dédie la dernière séquence à l'adoucissement et la subdivise en deux (d'abord la vénération, puis le pathos en direction des soldats).

Bilan de cette première analyse rhétorique : le contexte large (la sédition) et le contexte formel (l'identification de séquences contradictoires, tantôt tournées vers le reproche et la menace, et tantôt vers l'espoir et l'alliance) motivent la modélisation sur la conciliatio. Mais le contexte amont étroit (la conversion des soldats déjà opérée avant le discours) et l'ordre du discours (d'abord l'amertume, puis le miel) sont des points littéralement dysfonctionnels, en rupture avec le schéma du discours de réconciliation tel que nous l'avons vu précédemment; et en outre tout l'incipit, soit plus de la moitié du discours, est certes séparé du reste, séquencé, mais non identifié, non commenté, non modélisé.

Bref, la mise en forme par le modèle à la fois fonctionnel et formel de la conciliatio est loin d'aboutir à une prise en charge globale: nous dirons qu'elle est une hypothèse déduite d'une part du contexte et d'autre part du repérage d'un certain nombre de séquences paradoxales (menace versus adoucissement). Elle permet de les maintenir ensemble dans un même réseau et elle valide également à l'échelle supérieure un autre réseau de cohérence, entre le discours et la situation. Mais elle laisse de côté la question de l'ordo, de l'enchaînement inversé entre le doux et l'amer, de même qu'elle ne 
parvient pas à identifier la première séquence délimitée : c'est une séquence dont l'analyse ne nous dit pas à quoi elle sert, une séquence sans fonctionnalité (interne ou externe).

Autrement dit, les points de fragilité de cette analyse sont liés à la résistance des formes - que ce soient celles que l'on distingue mais dont on ne sait que faire, ou celles qui sont dysfonctionnelles au regard du fonctionnement normé retenu. Et ce sont également autant d'incitations à reformuler une autre hypothèse, à repenser un autre formatage et une autre identification générique.

41 Du point de vue méthodologique, nous dirons que la mise en forme rhétorique de l'énoncé est un opérateur de textualité (de tissage du texte) : le rhétoricien produit une forme possible du texte, actualise un texte possible, avec ses creux et ses reliefs, ses marges et sa cohérence. Et les limites ici répertoriées (incipit, ordre des séquences...) invitent alors à poursuivre l'enquête, à imaginer, produire, formaliser un autre texte possible. Nous le ferons avec l'analyse de Vossius.

\section{Deuxième mise en forme possible : un discours d'objurgation}

Vossius avait sérialisé sur le modèle de la conciliatio le premier discours de Germanicus, celui que Tacite se contente de résumer au style indirect : en revanche, il met le second discours qui nous occupe à présent en série avec un autre canevas, le genre discursif de l'objurgatio. C'est là un geste herméneutique décisif, mû par la prise en compte de deux éléments différents de contextualisation : d'abord le contexte étroit (les soldats émus) plutôt que le contexte large, actant en quelque sorte que la scène de parole n'est plus une scène de délibération (d'aide à la décision, si l'on veut!), mais une scène judiciaire, d'accusation et de reproches. Ce qui revient à dire que le chef d'armée n'en est plus à négocier l'apaisement, il en est à rendre la justice contre les fauteurs de troubles d'une part ; mais d'autre part ce chef d'armée, c'est Germanicus, que la tradition historique latine a doté d'un éthos de bonté et de dévouement exemplaires. Autrement dit les circonstances de la personne et les circonstances du temps fonctionnant en sens contraire (d'un côté la mansuétude, de l'autre l'heure de faire rendre des comptes), Vossius a alors tiré de son armoire à rhétorique le modèle d'un discours de réprimande compatible avec l'éthos de l'amitié, à savoir le discours d'objurgation.

L'objurgation est en effet l'une des déclinaisons possibles du genre judiciaire, proche de l'invective dans l'argumentation des reproches, mais antithétique dans l'éthos (benevolentia) et dans la proposition discursive (appel à se corriger) :

\footnotetext{
VOSSIUS. LE DISCOURS D'OBJURGATION ${ }^{24}$

Reste à voir les discours relevant du judiciaire : l'invective, l'objurgation, l'expostulatio [récrimination, reproche], l'exprobratio [condamnation sans appel] et la deprecatio [ou prière]. [...] L'objurgation est un discours de reproche, qui reprend une faute, mais qui se propose la correction de l'autre. Elle diffère de l'invective, en ce que celle-ci se porte presque toujours sur des ennemis et qu'elle a en vue ce qui nuit; celle-là s'attache à des amis et s'applique à ce qui est utile. Ajoutons que l'objurgation est la plupart du temps d'un supérieur (par ex. d'un empereur) à l'égard d'un soldat, d'un père à l'égard d'un fils.
} 
A partir de là, le canevas type de l'objurgatio est décliné : on commencera d'abord par l'exposé du crime, puis par son amplification; et on négociera une alternative à la fois fonctionnelle et formelle : pour les âmes dures, le discours finira sur une séquence de récrimination solennelle et/ou de menace; pour les âmes tendres, le discours commencera par une séquence dédiée à la manifestation de benevolentia :

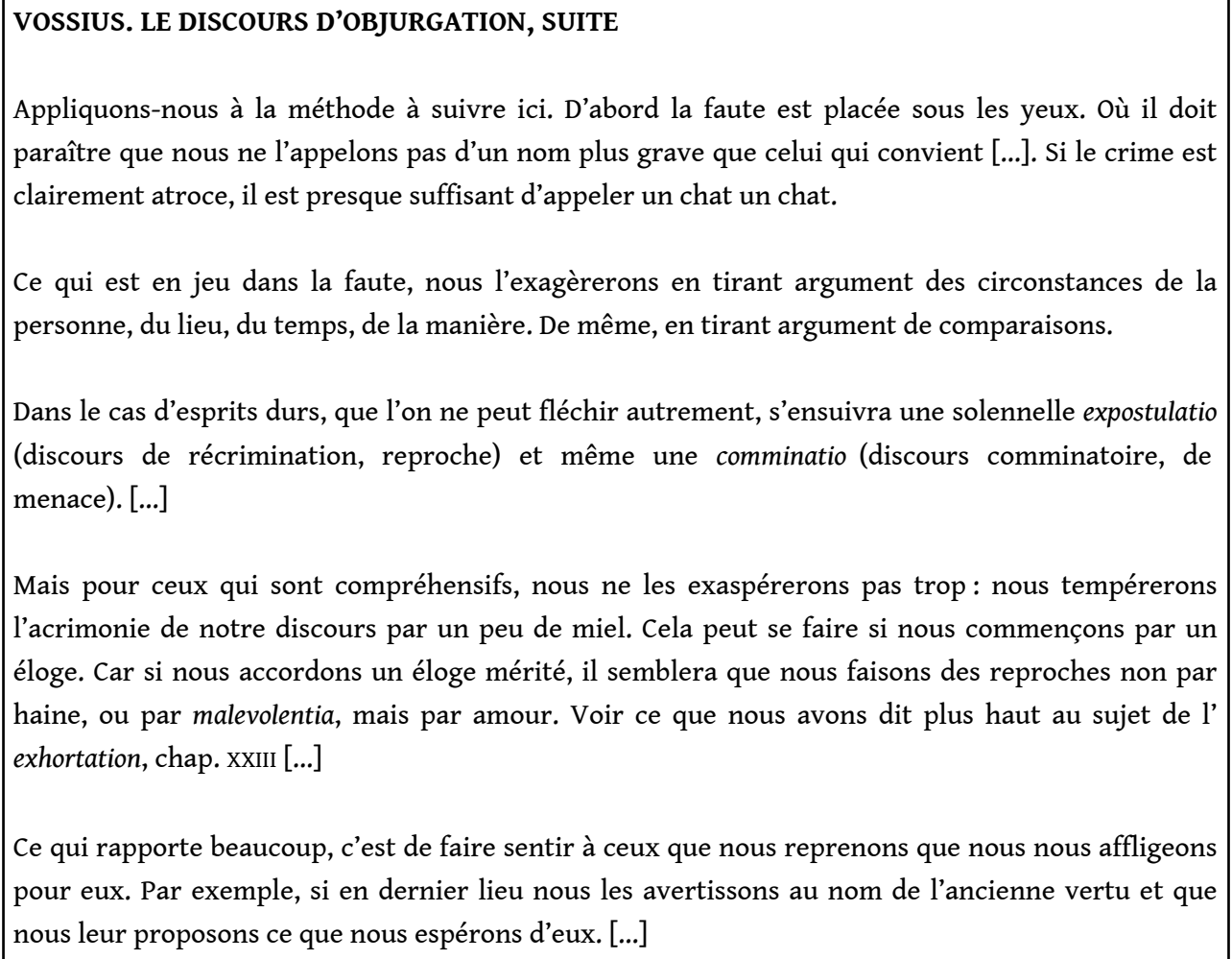

Reportons-nous à partir de là à l'analyse que fait Vossius du discours de Germanicus comme exemple standard d'objurgation, analyse où là aussi s'entremêlent les commentaires et les citations littérales (rappelons que nous mettons entre crochets les énoncés que le commentaire ne cite pas ${ }^{25}$ : 


\section{VOSSIUS. ANALYSE DU SECOND DISCOURS DE GERMANICUS : UNE OBJURGATION ${ }^{26}$}

[DESUNT : Ne croyez pas, dit-il, que mon épouse et mon fils me soient plus chers que mon père et la république. Mais mon père a pour sauvegarde sa propre majesté ; l'empire a ses autres armées. Ma femme et mes enfants, que j'immolerais volontiers à votre gloire, je les dérobe maintenant à votre fureur, afin que, si le crime ensanglante ces lieux, je sois la seule victime, et que le meurtre de l'arrière-petit-fils d'Auguste et de la belle-fille de Tibère n'en comble pas la mesure.]

Comme exemple de ce genre de discours, nous trouvons le discours de Germanicus à ses soldats rebelles. Voici quels sont les arguments concernant le genre de crime :

En effet, qu'y a-t-il eu pendant ces derniers jours que n'ait violé votre audace ? Quel nom donneraije à cette foule qui m'entoure ? Vous appellerai-je soldats? Vous avez assiégé comme un ennemi le fils de votre empereur; citoyens? Vous foulez aux pieds l'autorité du sénat: les lois même de la guerre, le caractère sacré d'ambassadeur, le droit des gens, vous avez tout méconnu, etc. [DESUNT : Jules César apaisa d'un mot une sédition de son armée, en appelant Quirites des hommes qui trahissaient leurs serments. Auguste, d'un seul de ses regards, fit trembler les légions d'Actium. Si nous n'égalons pas encore ces héros, nous sommes leurs rejetons; et l'on verrait avec surprise et indignation le soldat d'Espagne ou de Syrie nous manquer de respect.]

Puis il amplifie par un argument tiré des circonstances :

Et c'est la première légion, tenant les enseignes de Tibère ; c'est vous, soldats de la vingtième, compagnons de ses victoires, riches de ses bienfaits, qui payez votre général d'une telle reconnaissance!

S'ensuit une menace :

Voilà donc ce que j'annoncerai à mon père, qui de toutes les autres provinces ne reçoit que des nouvelles heureuses! [DESUNT : Je lui dirai que ses jeunes soldats, que ses vétérans, ne se rassasient ni de congés, ni d'argent ; qu'ici seulement les centurions sont tués, les tribuns chassés, les députés prisonniers ; qu'ici le sang inonde les camps, rougit les fleuves ; qu'ici enfin ma vie est à la merci d'une multitude furieuse. Pourquoi, le premier jour où j'élevai la voix, m'arrachiez-vous le fer que j'allais me plonger dans le cœur, trop aveugles amis? Il me rendait un bien plus généreux office, celui qui m'offrait son glaive: j'aurais péri du moins avant d'avoir vu la honte de mon armée. Vous auriez choisi un autre chef, qui sans doute eût laissé ma mort impunie, mais qui eût vengé le massacre de Varus et des trois légions. Car nous préservent les dieux de voir passer aux Belges, malgré l'empressement de leur zèle, l'éclatant honneur d'avoir soutenu la puissance romaine et abaissé l'orgueil de la Germanie!]

Après quoi il conclut. Voyez à ce propos comment il excite l'espoir et l'amour :

Âme du divin Auguste, reçue au séjour des immortels, image de mon père Drusus, mémoire sacrée d'un grand homme, venez, avec ces mêmes soldats, sur qui la gloire et la vertu reprennent leurs droits, venez effacer une tache humiliante, et tournez à la ruine de l'ennemi ces fureurs domestiques. [DESUNT: Et vous, dont je vois les visages, dont je vois les cœurs heureusement changés, si vous rendez au sénat ses députés, à l'empereur votre obéissance, à moi ma femme et mon fils, rompez avec la sédition, séparez de vous les artisans de trouble. Ce sera la marque d'un repentir durable, et le gage de votre fidélité.] 
La schématisation du canevas syntagmatique donnerait ici ceci, que nous mettons en regard du plan de Pelletier :

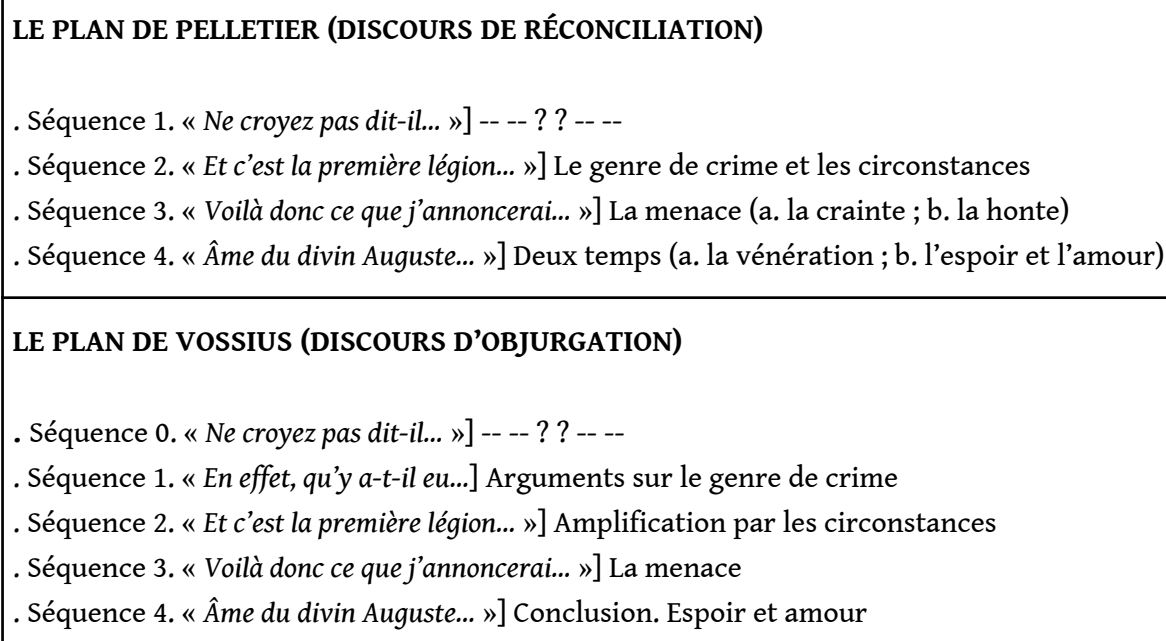

Commençons par cerner en quoi cette analyse se différencie de la précédente. Concernant le repérage de la proposition générale du discours tout d'abord, s'il s'agit d'une objurgatio, le dessein propositionnel (la séquence hiérarchiquement dominante du réseau) sera l'énoncé même de l'objurgation à se racheter, à bien faire, que l'on peut identifier une nouvelle fois dans la séquence ultime, ce sur quoi s'arrête précisément la citation littérale du texte tacitéen : "venez effacer une tâche humiliante, et tournez à la ruine de l'ennemi ces fureurs domestiques. » Second point, Vossius cite beaucoup moins de texte littéral que Pelletier : généralement il se contente de citer le début de chaque séquence, ponctuant occasionnellement la fin de l'extrait d'un «etc.». Et troisième point, Vossius retravaille l'ouverture: il en intègre une partie dans son commentaire, mais ne cite même pas l'énoncé incipitial du discours. Nous dirons qu'il distingue deux séquences, l'une " en creux », par son omission, l'autre, « en plein », par son identification.

Nous pouvons en venir dès lors aux ressemblances. Il est clair, tout d'abord, que malgré le changement du genre discursif (et malgré la divergence d'interprétation qu'il suppose concernant le contexte pragmatique), Vossius et Pelletier se retrouvent à peu près sur le séquençage, sur la répartition des masses du discours : trois séquences sont comparables (l'amplification du crime, la menace, la séquence finale dédiée à des affects positifs), même si deux des trois séquences sont moins finement analysées (la menace n'est pas subdivisée, pas plus que la conclusion) ; une séquence supplémentaire est intégrée à l'initiale parce qu'elle est délimitée (par l'embrayeur "en effet») et qu'elle est identifiée du point de vue de sa fonction dans l'argumentation (définition du genre du crime).

49 La fonctionnalité globale (l'objurgation) est donc compatible à la fois avec un canevas plus intégratif et avec le contexte situationnel étroit : elle l'emporte donc sur le canevas de la conciliatio, à ceci près que Vossius échoue lui aussi à rendre compte du dynamisme discursif - modélisant d'une part le texte sur un format type qui ne fait rien du tout début (d'où son éviction : il n'est même pas cité) et où la séquence d'adoucissement est censée être initiale et non finale. 
Bref, l'objurgation est une interprétation possible du discours, une identification relativement opérationnelle même si elle inclut elle aussi - peut-être forcément une part de reste, de résidus résistants ou dysfonctionnels. Comme toute mise en forme fixée, elle est autant une décision (d'en rester là) qu'une analyse, mais elle n'interdit pas une autre mise en forme possible, par exemple en recourant à la comminatio.

\section{Troisième mise en forme possible : un discours de menace}

Pourquoi aller chercher le genre du discours comminatoire ? Là aussi, la décision est liée à l'herméneutique du contexte, mais en se focalisant sur d'autres éléments que n'exploitent ni Pelletier ni Vossius : l'affect violent de Germanicus à l'orée du discours d'une part (discours d'un chef sous l'emprise de la fureur, nous dit Tacite ${ }^{27}$ ); et surtout la suite et les conséquences de son discours : le co-texte aval désigne ce discours comme un discours de condamnation sans appel (quoique justifiée : vera exprobrari ${ }^{28}$ ) et surtout il décrit le carnage qui s'ensuit, celui des chefs de la sédition par les soldats eux-mêmes, un carnage que je dirai expiatoire pour détourner la vraie menace d'une décimation plus grande, sur la légion entière. Or, sur la scène judiciaire, l'alliance du cinglant reproche d'ingratitude et de la menace s'incarne donc habituellement dans le genre du discours que l'on nomme comminatio:

\footnotetext{
PELLETIER. LE DISCOURS COMMINATOIRE OU DISCOURS DE MENACE ${ }^{29}$

Tout l'artifice de ce discours semble consister en ce que nous inspirions la crainte à celui que nous menaçons : c'est pourquoi la menace sera estimée d'autant meilleure que la crainte sera suscitée plus efficacement. Aussi le début doit-il être très abrupt, comme s'il était produit à l'improviste.

En outre, il convient que le genre de tout le discours soit concis, véhément (concitatus ${ }^{30}$ ), menaçant, plein de formules et de maximes impressionnantes, en rien recherché ou puéril. Toute la première Catilinaire peut être l'exemple absolu; elle a en effet un souffle véhément de part en part, et elle est efficace pour générer les sentiments les plus forts.

Doit être employée une amplification véhémente du crime ou du forfait contre lequel est requise la punition (supplicium) : ce qui peut donner lieu à un développement un peu long. [...]

Afin que le mouvement ne retombe pas, languide et froid, il faut recourir à la communicatio ${ }^{31}$ et avancer avec précaution, comme le fait Cicéron dans le passage loué.

Il sera de plus utile de décrire la chose assez clairement et dans ses conséquences désavantageuses à venir, de sorte qu'elle ne semble pas être dite, mais placée sous les yeux : ce qui est facile à suivre en imitant Cicéron, première Verrine [...].

La menace peut être conclue sous la forme d'une optatio [figure de pensées inverse de l'imprecatio: expression d'un désir, sous forme exclamative] et d'une exhortation, de façon que le discours ne semble pas être proféré sous l'emprise de la haine et de la malevolentia [...] : et ce, afin de ne pas s'aliéner les auditeurs et de faire en sorte qu'ils ne se détournent pas.
} 
Comme on le voit, le discours comminatoire partage avec la conciliation et l'objurgation l'alliance de séquences contrastées (de réprimande versus d'adoucissements) mais il est le seul canevas type qui nous permette de prendre en compte deux éléments qui nous avaient résisté, le tout début (« Aussi le début doit-il être très abrupt, comme s'il était produit à l'improviste») et la position ultime de la séquence d'adoucissement («La menace peut être conclue [...] de façon que le discours ne semble pas être proféré sous l'emprise de la haine et de la malevolentia»). Il nous permet en outre quelques réglages fins dans le formatage des séquences intermédiaires, en termes de figures et pas seulement en termes de schémas argumentatifs.

Prendre l'hypothèse d'un dispositif comminatoire permet alors de mettre en place une autre version possible du discours de Germanicus, plus finement formatée et fonctionnalisée : ce sera une ultime hypothèse de formalisation, que je placerai donc sous l'égide de ces outils rhétoriques anciens que sont les genres du discours, mais que je mènerai en l'occurrence seule puisqu'aucun rhétoricien n'a pris le second discours de Germanicus comme exemple de comminatio.

54 Je procèderai cependant comme mes prédécesseurs. J'identifierai tout d'abord comme séquence focale l'énoncé littéral de la menace (que l'on trouve ici en position centrale et non pas ultime) : «Voilà donc ce que j'annoncerai à mon père [l'empereur Tibère luimême, père adoptif de Germanicus]: [...] qu'ici enfin ma vie est à la merci d'une multitude furieuse.»Et je modéliserai ensuite les séquences en reprenant les outils descriptifs répertoriés à la fois par le genre de la comminatio et par ceux de la conciliatio ou de l'objurgatio, puisqu'au demeurant l'identification formelle des séquences est de part et d'autre relativement stable quel que soit le contexte fonctionnel retenu : 


\section{NOILLE. ANALYSE DU SECOND DISCOURS DE GERMANICUS : UN DISCOURS DE MENACE}

Début ex abrupto et véhément comme indiqué dans le canevas du discours comminatoire. Plus précisément, invectiva sous la forme véhémente d'une prolepsis, d'une réponse à une objection implicite :

Ne croyez pas, dit-il, que mon épouse et mon fils me soient plus chers que mon père et la république. Mais mon père a pour sauvegarde sa propre majesté ; l'empire a ses autres armées. Ma femme et mes enfants, que j'immolerais volontiers à votre gloire, je les dérobe maintenant à votre fureur, afin que, si le crime ensanglante ces lieux, je sois la seule victime, et que le meurtre de l'arrière-petit-fils d'Auguste et de la belle-fille de Tibère n'en comble pas la mesure.

Arguments concernant le genre de crime : tirés d'une enumeratio, d'un détail des griefs, et ce, sous la forme véhémente d'une communicatio (d'une prise à partie du destinataire), comme indiqué dans le canevas de la comminatio :

En effet, qu'y a-t-il eu pendant ces derniers jours que n'ait violé votre audace ? Quel nom donneraije à cette foule qui m'entoure? Vous appellerai-je soldats? Vous avez assiégé comme un ennemi le fils de votre empereur; citoyens? Vous foulez aux pieds l'autorité du sénat: les lois même de la guerre, le caractère sacré d'ambassadeur, le droit des gens, vous avez tout méconnu.

Amplification du crime: argument tiré d'une comparaison a simili (comme indiqué dans le canevas de l' objurgatio) :

Jules César apaisa d'un mot une sédition de son armée, en appelant Quirites des hommes qui trahissaient leurs serments. Auguste, d'un seul de ses regards, fit trembler les légions d'Actium. Si nous n'égalons pas encore ces héros, nous sommes leurs rejetons; et l'on verrait avec surprise et indignation le soldat d'Espagne ou de Syrie nous manquer de respect.

Amplification du crime, suite : argument tiré des circonstances de la personne :

Et c'est la première légion, tenant les enseignes de Tibère ; c'est vous, soldats de la vingtième, compagnons de ses victoires, riches de ses bienfaits, qui payez votre général d'une telle reconnaissance!

Énoncé de la menace : ressort du pathos de la crainte

Voilà donc ce que j'annoncerai à mon père, qui de toutes les autres provinces ne reçoit que des nouvelles heureuses! Je lui dirai que ses jeunes soldats, que ses vétérans, ne se rassasient ni de congés, ni d'argent; qu'ici seulement les centurions sont tués, les tribuns chassés, les députés prisonniers; qu'ici le sang inonde les camps, rougit les fleuves ; qu'ici enfin ma vie est à la merci d'une multitude furieuse.

Menace, suite : ressort du pathos de la honte. Récrimination solennelle envers ceux dont on veut cependant le bien, c'est-à-dire expostulatio (variante possible de la comminatio dans le canevas de l'objurgation) :

Pourquoi, le premier jour où j'élevai la voix, m'arrachiez-vous le fer que j'allais me plonger dans le cœur, trop aveugles amis? Il me rendait un bien plus généreux office, celui qui m’offrait son glaive : j'aurais péri du moins avant d'avoir vu la honte de mon armée. Vous auriez choisi un autre chef, qui sans doute eût laissé ma mort impunie, mais qui eût vengé le massacre de varus et des trois légions. Car nous préservent les dieux de voir passer aux Belges, malgré l'empressement de leur zèle, l'éclatant honneur d'avoir soutenu la puissance romaine et abaissé l'orgueil de la Germanie!

Conclusion: conformément au canevas du discours comminatoire, éthos d'un homme sans malevolentia, Exercices de rhétorique 3 d201'Al'invocation à Auguste : ressorts de l'espoir) puis par une exhortation aux soldats (ressorts de l'amour) :

Âme du divin Auguste, reçue au séjour des immortels, image de mon père Drusus, mémoire sacrée d'un grand homme, venez, avec ces mêmes soldats, sur qui la gloire et la vertu reprennent leurs 
Ce qui nous donne comme schéma syntagmatique (en parallèle avec les précédents) :

\author{
LE PLAN DE PELLETIER (DISCOURS DE RÉCONCILIATION) \\ . Séquence 1. « Ne croyez pas dit-il... »] -- -- ? ? -- -- \\ . Séquence 2. "Et c'est la première légion... »] Le genre de crime et les circonstances \\ . Séquence 3. « Voilà donc ce que j'annoncerai... »] La menace (a. la crainte ; b. la honte) \\ . Séquence 4. «Âme du divin Auguste... »] Deux temps (a. la prière à Auguste et l'espoir ; b. l'adresse \\ aux soldats et l'amour)

\section{LE PLAN DE VOSSIUS (DISCOURS D'OBJURGATION)} \\ . Séquence 0. « Ne croyez pas dit-il... »] -- -- ? ? -- -- \\ . Séquence 1. «En effet, qu'y a-t-il eu...] Arguments sur le genre de crime \\ . Séquence 2. «Et c'est la première légion... »] Amplification par les circonstances \\ . Séquence 3. "Voilà donc ce que j'annoncerai... »] La menace \\ . Séquence 4. « Âme du divin Auguste... »] Conclusion : a. l'espoir et b. l'amour

\section{LE PLAN DE NOILLE (DISCOURS DE MENACE)} \\ . Séquence 0. «Ne croyez pas dit-il... »] Début ex abrupto sous la forme d'une prolepse \\ . Séquence 1. «En effet, qu'y a-t-il eu...] Argument sur le genre de crime tiré d'une énumération et \\ sous la forme d'une communicatio \\ . Séquence 2.a. «Jules César apaisa d'un mot...] Amplification par une comparaison \\ . Séquence 2.b. «Et c'est la première légion... »] Amplification par les circonstances \\ . Séquence 3.a. « Voilà donc ce que j'annoncerai... »] La menace : a. pathos de la crainte \\ . Séquence 3.b. « Pourquoi, le premier jour... »] La menace, suite : b. pathos de la honte \\ . Séquence 4.a. "Âme du divin Auguste... »] Conclusion. Ressorts de la benevolentia : a. une optatio \\ (espoir) \\ . Séquence 4.b. «Et vous, dont je vois les visages...] Conclusion, suite : b. une exhortation (amour)
}

Disons que c'est là la modélisation qui est la plus intégrative et dont la granularité est la plus fine : le discours de Germanicus est devenu par la division et l'ordonnancement que j'ai opérés un exemple presque standard d'une menace en forme. Mais cette modélisation oblige en revanche à rompre avec l'image conventionnelle de bonté, avec l'éthos exemplaire d'amitié et de popularité construit autour du personnage de Germanicus. Ce qui n'est pas rien: le discours ainsi formaté s'avère - à l'interprétation - globalement incompatible avec son énonciateur. Il s'agit bien évidemment d'une faiblesse majeure pour ce qui est - à tout prendre - simplement une troisième analyse rhétorique de l'énoncé, une troisième mise en forme possible, une troisième modélisation textuelle.

On pourrait ajouter d'autres formalisations possibles - on en ajoutera sans doute d'autres : il suffit pour cela de raffiner notre compétence modélisatrice (en l'adossant par exemple à une technique méthodique de séquençage, de mise en réseau et de formalisation dynamique du rythme, du déroulé sémantique); d'enrichir également notre encyclopédie des formes type de séquences et des canevas type du discours; enfin de complexifier la standardisation du cas en exploitant et en interprétant tel ou tel autre circonstant contextuel. 
Mais c'est déjà anticiper sur les quelques conclusions méthodologiques que nous nous proposons de tirer de ce parcours.

\section{Les genres du discours : usages possibles}

\section{Une leçon de structuration}

59 La première leçon que l'on peut tirer de cette expérience de formalisation peut se dire très simplement : les genres du discours nous apprennent à structurer des énoncés. Ce sont des modèles formels qui servent de médiation et de point d'appui à notre compétence formalisatrice en lui offrant, sur un texte donné, des hypothèses possibles de mise en forme sémantiquement pertinente.

Dans le détail de l'expérience, nous avons vu tout d'abord que la structuration opérait à différents niveaux (le tout, la partie) et selon différentes modalités (gestion de la division, du regroupement, de la succession) :

- les genres du discours nous autorisent tout d'abord à postuler, par principe, une unité globale de sens et de forme (fût-elle complexe, hybride, oblique) ; autrement dit, ils nous permettent de considérer le tout du discours comme susceptible de division et de partition ;

- les genres du discours nous apprennent à repérer, délimiter et identifier des séquences; et le séquençage des parties reste absolument stable d'un modèle global à un autre : c'est donc là un acquis fondamental, que de nous permettre ainsi de transformer le tissu textuel d'un discours écrit ${ }^{32}$, donné à la relecture, en un composé mosaïque ;

- les genres du discours nous expliquent comment mettre en réseau les séquences délimitées, dans un canevas hiérarchisé ;

- les genres du discours nous proposent une grille d'interprétation concernant l'identité globale du discours, sa séquence focale ;

- les genres du discours enfin nous permettent d'interroger les processus liés à la dynamique et à l'enchaînement des séquences.

Il s'agit là d'un système régulateur modulaire, nous donnant les moyens de structurer la masse (l'extension), le multiple (le disparate), le successif (le cumulatif).

\section{Une leçon d'interprétation}

La seconde leçon corrélée est que les genres du discours nous placent devant un choix herméneutique : soit recenser les formes possibles d'un discours et en rester au niveau de l'analyse, soit nous engager dans une des hypothèses et stabiliser en conséquence toute la structure au service d'une unité sémantiquement pertinente.

63 En effet, si, comme nous l'avons vu avec l'exemple de Germanicus haranguant ses soldats, il y a un relatif consensus sur la mise en forme, le repérage et l'autonomisation des séquences et une grande stabilité sur leur identification rhétorique, la discussion commence avec les décisions herméneutiques concernant d'une part la focalisation (la hiérarchie des séquences) et d'autre part la modélisation syntagmatique et paradigmatique du discours entier. Et nous venons précisément de faire l'expérience à la fois de l'évidence et de la résistance des formes dans un cadre encyclopédique donné : de leur évidence tout d'abord pour une compétence formalisatrice experte (telle que celle de la technique rhétorique); et de leur résistance ensuite à perdre leur 
identité propre, leur visibilité (leur perceptibilité), à être normalisées (dissoutes) dans une modélisation fonctionnelle ad hoc.

Libre alors à l'herméneute de faire le choix de les conserver, de les gauchir ou de les ignorer: si la rhétorique travaille dans le remarquable et le sériel, dans l'exemple, la critique est dans la décision et a la responsabilité de son point de perspective.

\section{Un art de lire général ?}

Il est clair que dans les pratiques historiquement attestées de l'ancienne rhétorique, les genres du discours ont été orientés, par-delà l'apprentissage qui en était fait dans la relecture guidée et l'annotation d'une bibliothèque, vers la production d'autres discours. Les genres du discours ne sont pas une ressource pour monumentaliser un texte, mais pour le rendre productif — imitable car sérialisable, transformable car pluralisable.

De toute évidence, dans notre propre culture du texte et de l'interprétation, les genres du discours ne sont pas d'emblée opérants à ce niveau-là de la production discursive, mais ils fournissent en amont une grille de formalisation et d'interprétation pour un certain nombre de textes. Lesquels précisément ? Là est le cœur du problème.

Il semble y avoir tout d'abord une correspondance entre l'outillage théorique et un corpus historiquement situé, contemporain de l'outillage, les discours écrits de l'antiquité au XIX ${ }^{e}$ siècle: une forme isolable et analysable, le discours, qu'elle soit assemblée dans des recueils, insérée et commémorée dans les ouvrages des historiens, densifiée et exhibée au théâtre, modulée et adaptée dans les échanges romanesques. Et ce gain-là n'est pas à négliger. Qu'une pratique de formalisation rejoigne des pratiques d'écriture et de lecture qui lui soient contemporaines a l'avantage de la vraisemblance... toujours en décalage avec l'outillage théorique tel que, somme toute, nous l'avons fait émerger au présent, dans notre propre actualité, à partir du traitement complexe et sophistiqué que nous avons fait subir aux discours rhétoriques anciens. Nous pouvons décrire et interpréter les discours de l'Énéide ainsi, tout comme ceux de Chateaubriand: qui ne voit l'artifice, du point de vue de l'historien? À moins de poser l'existence de structures anthropologiques de l'argumentation, qui nous permettent de valider la démarche, de Virgile à Malraux, et d'Obama à Cicéron. On le peut, que d'autres le fassent...

Nous préférons pour notre part nous en tenir à la position suivante: nous considérerons que la validité de la démarche est d'abord méthodologique. Pouvoir décrire avec le relais de modèles de schématisation (les genres du discours) ce qui s'éprouve dans la relecture comme un énoncé discursif textualisé (tissé), à la fois long, disparate et dynamique, tel est le résultat le plus fiable, et le plus humble - ne nous permettant pas de nous prononcer ni sur les généalogies ni sur les intentions, ni sur l'humain ni l'Histoire. Un savoir-lire local, en quelque sorte, portant sur le texte des discours, mais qu'il est possible d'utiliser comme matrice pour nous permettre de penser des opérations plus générales de structuration textuelle, par division, par cohésion, par transition ${ }^{33}$... 
70 Reste un dernier massif d'interrogations dont nous ne savons trop que faire: où commence (et où s'arrête) un tel objet localisé - le texte d'un énoncé discursif étendu, disparate et dynamique ${ }^{34}$ ? Une fois qu'on a mis à part tout ce qui était discours entier, étendu d'un seul bloc de son entame archétypale ("Mes chers compatriotes... ») à sa péroraison («... vive la République et vive la France !»), où émerge et se perd un énoncé discursif ${ }^{35}$ ? Tout énoncé est-il un syntagme discursif? Tout énoncé est-il susceptible d'être globalisé et morcelé, pluralisé et relié ? Tout énoncé - narratif, dramaturgique, poétique - a-t-il vocation à être un énoncé discursif mosaïque ? Et les composés composites que sont les genres du discours peuvent-ils ressaisir, dans la polyphonie discursive et l'émiettement énonciatif des énoncés innombrables qui meublent nos vies, ceux de la conversation comme ceux de la narration, ceux de la vie quotidienne come ceux de la littérature, les particules élémentaires de leur syntaxe discursive?

71 Nous avouerons volontiers qu'entre la modélisation structurale initiée par la pratique des genres du discours et une modélisation générale de la structuration syntagmatique des textes, telle qu'on peut la déduire de catégorisations a priori, il nous manque encore des modèles empiriques qui nous permettent d'expérimenter, sur le terrain du roman en particulier, la variété énonciative en continuité avec la variation discursive.

Paris, mai 2014.

\section{NOTES}

1. Depuis le De conscribendis Epistolis d'Érasme en effet (1521), la typologie des epistolae se calque sur celles des orationes, avec variantes et adaptations au contexte privé tout autant qu'à la communication in absentia.

2. Dans Exercices de rhétorique 1 | 2013, URL: http://rhetorique.revues.org/122.

3. Dans Exercices de rhétorique 2 | 2013, URL: http://rhetorique.revues.org/176.

4. Nous dressons ici la liste exhaustive des discours, à partir de deux listes, celle de Vossius et celle de Pelletier. Voir G. J. Vossius, Rhetorices contractae, sive Partitionum Oratoriarum libri quinque ( $1^{\text {re }}$ éd. Leyde, 1621), Saumur, 1677 ; et G. Pelletier, Reginae Palatium Eloquentiae ( $1^{\text {re }}$ éd. Paris, 1641), Cologne, 1709. Dans l'article cité en note 1, F. Goyet ne retient pas tous les items.

5. Sur les listes de buts oratoires institués, auxquels correspondent à chaque fois un discours spécifié, voir F. Goyet, op. cit. Ce sont des listes très stables, de l'antiquité au XIXe siècle. Ce qu'il faut bien comprendre, c'est que derrière un mot tel que "se plaindre ", il n'y a pas de la psychologie, il y a de la technique, des marqueurs qui peuvent fonctionner comme embrayeurs descriptifs autant que comme propositions prescriptives. Un relais utile pour mieux apprécier ces listes est la paraphrase semi-technique qu'en fait Charles Batteux (Cours de Belles Lettres, Paris, Dessaint et Saillant, t.IV, 1753, p. 271) : «Le genre épistolaire n'est autre chose que le genre oratoire rabaissé jusqu'au simple entretien ; par conséquent il y a autant d'espèces de lettres qu'il y a de genres d'oraisons. On conseille dans une lettre, on détourne, on exhorte, on console, on demande, on recommande, on réconcilie, on discute : et alors on est dans le genre délibératif. On accuse, on se plaint, on menace, on demande que les torts soient réparés: c'est le genre judiciaire. On loue, on blâme, on raconte, on félicite, on remercie, etc.: c'est le genre démonstratif. » 
6. Sur ce massif des études contemporaines, nous ne ferons que renvoyer aux deux écrits fondateurs de notre contemporanéité que sont d'une part l'ouvrage de V.N. Volochinov, Marxisme et philosophie du langage, éd. P. Sériot et I. Tylkowski, Limoges, éd. Lambert-Lucas, 2010 ; et d'autre part M. Bakhtine, Esthétique de la création verbale, Paris, Gallimard, 1984, « Les genres du discours ", p. 284 sq.

7. Sur la notion de texte, voir infra note 32.

8. Voir P. Fontanier, Les Figures du discours, Paris, Flammarion, 1977, p. 433 : « Outre les figures de pensées qui font l'objet des paragraphes précédents, presque tous les rhéteurs, en distinguent beaucoup d'autres : la Commination, l'Imprécation, l'Optation, la Déprécation, le Serment, la Dubitation, la Licence. Mais ce ne sont, très-probablement des figures d'aucune espèce. » Et un peu plus loin, en reprenant la liste standard des «buts» discursifs (preuve qu'il la connaissait), Fontanier poursuit (ibid., p. 435) : "Mais alors, autant de nouvelles figures, que de sentiments ou de passions diverses [...]. Alors, dis-je, l'injure, le reproche, le blâme, la louange, la flatterie, le compliment, le conseil, l'exhortation, l'offre, la demande, le remerciement, la plainte, et que saisje encore (sic), seront autant de figures qu'il faudra classer [...] ?»

9. Sur ce point, voir $\mathrm{Ch}$. Noille, «Rhétorique de la périphrase : la figure comme dispositif de mise en forme », dans J. C. Cavallin et J. D. Mazaré, Le Mot propre et la périphrase, Paris, Garnier, 2014, p. 27-43.

10. Pour reprendre la formule de Michel Charles dans son Introduction à l'étude des textes, Paris, Le Seuil, 1995, p. 117.

11. L'analyse de cas reprendra le travail que nous avons mené pour une communication donnée au colloque international «Usages et fonctions de la rhétorique» (Bruxelles, mai 2013) sous le titre : « Form Follows What ? L'exemple d'un discours politique »; pour une première version écrite de cette étude de cas, voir notre article " Rhétorique de la lecture et compétence formalisatrice » dans l'Atelier Fabula, URL : http://www.fabula.org/atelier.php? Rhetorique_de_la_lecture_et_competence_formalisatrice.

12. Tacite, Annales, livre I, ch. 31-49, révolte des légions de Germanie, trad. J. L. Burnouf, Paris, Hachette, 1859. Pour une investigation sur les analyses rhétoriques menées sur d'autres discours dans les Annales, voir I. Cogitore, «Analyse rhétorique pratique de quelques discours des Annales de Tacite » dans La Revue des Études latines 90 (2012), p. 282-295.

13. G. J. Vossius, Rhetorices contractae, op. cit.

14. G. Pelletier, Reginae Palatium Eloquentiae, op. cit.

15. Souvenir de Raymond Queneau, Les Fleurs bleues, Paris, Gallimard, 1965, incipit : « Le vingtcinq septembre douze cent soixante-quatre, au petit jour, le duc d'Auge se pointa sur le sommet du donjon de son château pour y considérer, un tantinet soit peu, la situation historique. Elle était plutôt floue. Des restes du passé traînaient encore çà et là, en vrac. Sur les bords du ru voisin, campaient deux Huns; non loin d'eux un Gaulois, Éduen peut-être, trempait audacieusement ses pieds dans l'eau courante et fraîche. Sur l'horizon se dessinaient les silhouettes molles de Romains fatigués [...]. Quelques Normands buvaient du calva. »

16. Tacite, op cit., I, 34 sq.

17. G. J. Vossius, Rhetorices contractae, op. cit., II, 22, p. 188-192, trad. L. Vianès dans Exercices de rhétorique, 1 | 2013, URL : http://rhetorique.revues.org/170.

18. G. J. Vossius, Rhetorices contractae, II, 22, op. cit.

19. Tacite, op. cit., I, 40 sq.

20. G. J. Vossius, Rhetorices contractae, II, 22, op. cit. Repris textuellement dans G. Pelletier, Reginae Palatium Eloquentiae, «Artificium Conciliationis » [« L'art du discours de réconciliation »], op. cit., p. 874-876.

21. Comparer Tacite, op.cit., I, 41 (co-texte amont) : «Ils se jettent au-devant d'Agrippine, la supplient de revenir, de rester; et, tandis qu'une partie essaye d'arrêter ses pas, le plus grand nombre retourne vers Germanicus. Lui, encore ému de douleur et de colère, s'adressant à la foule 
qui l'environne [...].» Et Tacite, ibid., I, 44 (co-texte aval) : «Touchés par ce discours, ils lui demandent grâce (supplices), et, reconnaissant la vérité de ses reproches, ils le conjurent de punir le crime, de pardonner à l'erreur, et de les mener à l'ennemi [...]. »

22. Tacite, ibid., I, 42-43 in extenso, sans le paragraphage moderne.

23. G. Pelletier, Reginae Palatium Eloquentiae, op. cit., p. 874-876, trad. F. Goyet et Ch. Noille. Nous mettons en italiques le commentaire proprement dit; en caractères romains, les citations ; entre crochets, les énoncés de Tacite que ne cite pas Pelletier.

24. G. J. Vossius, Rhetorices contractae, op. cit., II, 16, p. 211-215, trad. Ch. Noille.

25. Pour les conventions typographiques que nous reprenons ici, voir la note 23.

26. G. J. Vossius, op. cit., II, 16, trad. Ch. Noille.

27. Voir Tacite, op.cit.: "Lui, encore ému de douleur et de colère, s'adressant à la foule qui l'environne [...]. »

28. L'exprobratio ou discours de condamnation sans appel est l'un des deux discours d'accusation institutionnalisé hors tribunaux (avec l'expostulatio ou plainte, récrimination, reproche). Si nous n'avons pas retenu ici ce genre pour analyser le tissu du second discours de Germanicus, c'est que nous sommes partis sur des modèles susceptibles d'intégrer les séquences paradoxales de l'adoucissement et de la menace, alors que l'exprobratio exclut la présence de tout miel. Sur l' expostulatio et l'exprobratio, voir G. J. Vossius, «Les discours de reproches et de condamnation sans appel », éd. et notes de F. Goyet, trad. L. Vianès, dans Exercices de rhétorique 2|2013, URL : http://rhetorique.revues.org/190.

29. G. Pelletier, Reginae Palatium, « Comminationis artificium » ["L'art du discours de menace »], p. 944-945, trad. Ch. Noille.

30. Par la véhémence, la comminatio entre en résonance avec la concitatio ou discours de sédition. Sur la concitatio, voir G. J. Vossius, Rhetorices contractae, op. cit., II, 22, éd. et notes F. Goyet, trad. L. Vianès, op. cit.

31. Figure de pensées, qui consiste à simuler qu'on demande l'avis du destinataire. Voir P. Fontanier, Les Figures du discours, op. cit., p. 414 : «Par la Communication, afin de mieux persuader ceux à qui ou contre qui l'on parle, et même souvent afin de leur arracher des aveux plus ou moins pénibles, on a l'air de les consulter, d'entrer en conférence avec eux, et de s'en rapporter à ce qu'ils décideront eux-mêmes. »

32. J'utilise donc le mot texte non pas dans le sens d'un énoncé autonomisé, coupé des contextes, mais dans le sens d'un énoncé relié à son contexte de mise en forme, d'un énoncé tissé, bref d'un énoncé lisible car articulé, au sens hjemslévien et genettien où la forme ne s'oppose pas au contenu, mais à la substance, où la forme, précisément, articule le sens. Voir G. Genette, « Raisons de la critique pure » (1966), Figures II, Le Seuil, 1969, coll. « Points », 1979, p. 19.

33. Ce sont là les trois opérations que nous travaillons dans l'article précédent que nous avons consacré à la rhétorique comme art d'analyser les discours («Le discours d'un Prince [Énéide I, 597-610]. Archéologie de la disposition », op. cit.).

34. Là commence aussi la réflexion des linguistes russes, de Volochinov à Bakhtine...

35. En jeu ici, entre autres, la définition de la tirade et de la scène, dans leur rapport à l'unité discursive. 


\section{AUTEUR}

\section{CHRISTINE NOILLE}

Université Grenoble Alpes - RARE Rhétorique de l'Antiquité à la Révolution 